\title{
Do U/Pb-SHRIMP Dating and Pb Stepwise Leaching (PbSL) Analyses Confirm the Lack of Precambrian Basement Outcrops in Thailand?
}

\author{
Bent T. Hansen ${ }^{1 *}$, Klaus Wemmer ${ }^{1}$, Prinya Putthapiban², Ilka C. Kleinhanns ${ }^{1,3}$, \\ Franziska Wilsky ${ }^{1}$ \\ ${ }^{1}$ Geoscience Centre of the Georg-August-University, Göttingen, Germany \\ ${ }^{2}$ Geoscience Programme, Kanchanaburi Campus, Mahidol University, Tambon Salaya, Thailand \\ ${ }^{3}$ Department of Geoscience, University of Tuebingen, Tübingen, Germany \\ Email: ${ }^{*}$ bhansen@gwdg.de
}

Received 24 August 2014; revised 20 September 2014; accepted 15 October 2014

Copyright (C) 2014 by authors and Scientific Research Publishing Inc.

This work is licensed under the Creative Commons Attribution International License (CC BY).

http://creativecommons.org/licenses/by/4.0/

(c) (i) Open Access

\begin{abstract}
$\mathrm{U} / \mathrm{Pb}$-SHRIMP dating and Pb stepwise leaching (PbSL) experiments on zircons and garnets, respectively have confirmed the lack of Precambrian outcrops within the crystalline basement of Thailand. The obtained data for the high grade metamorphism show Indosinian ages ranging from 225 - $200 \mathrm{Ma}$ as previously suggested for the vast majority of outcrops in NW-Thailand ([1] and references therein), as well as a small group of ages in the range of $445 \mathrm{Ma}$ in the Lampang Province. Further, the age of a thermal imprint around 60 Ma was confirmed near Surat Thani, Peninsular Thailand, and only a few indications of older ages for the unknown source areas were detected in detrital components.
\end{abstract}

Keywords

SHRIMP Dating, Pb Stepwise Leaching (PbSL), Zircons, Basement, Thailand

\section{Introduction}

In 1939, [2] were the first to publish a description on the occurrence of gneisses in Thailand. These high-grade

${ }^{*}$ Corresponding author.

How to cite this paper: Hansen, B.T., Wemmer, K., Putthapiban, P., Kleinhanns, I.C. and Wilsky, F. (2014) Do U/Pb-SHRIMP Dating and $\mathrm{Pb}$ Stepwise Leaching (PbSL) Analyses Confirm the Lack of Precambrian Basement Outcrops in Thailand? Open Journal of Geology, 4, 505-517. http://dx.doi.org/10.4236/ojg.2014.410037 
metamorphic rocks are generally overlain by fossiliferous sedimentary rocks of all periods of phanerozoic strata. Consequently, [3] [4] assigned a Precambrian age to all gneisses and also to all weakly metamorphosed rocks in West Thailand. In a more differentiated approach, [5] kept the Precambrian age for the gneisses, while weaker metamorphic sedimentary strata was considered as being Paleozoic. During the 1990s, isotopic dating of gneisses belonging to the so called "Precambrian basement" showed that these gneisses were formed during a high-grade metamorphic event in Upper Triassic time (for a comprehensive discussion see [1] and references herein). However, as upper intercept ages of zircons and Nd-model ages yield Precambrian ages for the precursors of the gneisses, the existence of a Precambrian basement within Thailand is still debated among geologists. For this reason, further investigations by means of SHRIMP analyses of zircons and PbSL dating of syn-metamorphic garnets were undertaken at selected locations. This study presents the first set of U/Pb SHRIMP data of regional importance for the high-grade metamorphic crystalline basement of Thailand. Up to now, modern $\mathrm{U} / \mathrm{Pb}$ geochronology was spatially restricted to the Khlong Marui shear zone ([6], LA-ICP-MS), to granitoids in N and SW Thailand ([7], ion microprobe) and to detrital zircons from a mélange within the Inthanon zone of N-Thailand ([8], LA-ICP-MS).

\section{General Geology of the Thai Basement Rocks}

The inferred Precambrian high-grade metamorphic rocks of amphibolites-facies are widely exposed in Northern Thailand and occur sporadically in the Western, Eastern and Southern Peninsula of the country (Figure 1). According to [1] [9] [10], the evolution of the so called "Precambrian basement" in Thailand can be described as follows.

General rock sequences typical for the crystalline basement areas are mainly granitic to granodioritic paragneisses, mica schists, hornblende schists and amphibolites. These rocks are often associated with orthogneisses, migmatites, granites, pegmatites and aplites. The crystalline basement rocks appear often as elongate bodies in a more or less N-S direction ([11] [12]). They are most often in contact with sedimentary rocks of Paleozoic age and greenschistfacies metamorphism. Younger sediments trend to weaker metamorphic or diagenetic grades. An unconformity between basement and cover rocks was proposed due to the jump in metamorphism from amphibolite to greenschistfacies metamorphic conditions ([10]), even so the contact itself is not exposed. [1] [13]-[16] assigned an Upper Triassic age for the last high-grade metamorphism of regional character in most parts of the basement. Corresponding P-T conditions of $3.5-4 \mathrm{~Kb}$ pressure and $650^{\circ} \mathrm{C}-660^{\circ} \mathrm{C}$ were reported by ([10]). The protolithes of the so called "Precambrian basement" can be considered as a former sequence of impure arenaceous to argillaceous sediments with mainly impure carbonate before the regional metamorphism. The age of the original sedimentary rocks before metamorphism was investigated by the $\mathrm{Rb} / \mathrm{Sr}$ small slab technique (after [17]) gave a maximum depositional age of around $600 \mathrm{Ma}$ [15]. Subsequent following HT metamorphism events of localized character were reported from several places in Thailand and will be discussed below.

\section{Results}

In order to test the validity of the U/Pb ages obtained by zircon multigrain analyses, U/Pb-SHRIMP dating on zircons from four selected localities and $\mathrm{Pb}$ stepwise leaching (PbSL) on one garnet separate were performed.

\subsection{Th04-02. The Muang Pan Gneiss, N-Thailand ( $\left.18^{\circ} 39^{\prime} \mathrm{N} / 99^{\circ} 30^{\prime} \mathrm{E}\right)$}

The Muang Pan Gneiss is located on the eastern flank of the Northwestern basement complex, east of the Inthanon range or western region of the Lampang Province. The investigated sample originates from a strongly weathered muscovite-biotite gneiss from an outcrop some $12 \mathrm{Km}$ south of Amphoe Muang Pan in the Lampang area (Figure 1). The gneiss is overlain by phyllites. The transition from the phyllites into the gneiss is continuous and the foliation of the gneiss corresponds to the cleavage of the phyllites (125/78, steeply dipping to the SE).

[1] noted that a conventional U/Pbzircon age pointing to a Silurian intercept of $428 \pm 19$ Ma could be of importance for the evolution of the basement gneisses, therefore we selected this location for detailed investigations. Up to then, a comparable old age of $446 \pm 5 \mathrm{Ma}\left({ }^{40} \mathrm{Ar} /{ }^{39} \mathrm{Ar}\right.$ on amphiboles) was only reported once by [18] from the Langsang gneiss (equal to Larn Sang in our text) in the Wang Chao shear zone where apart from that only Mid-Oligocene ages had been observed ([14] [18]).

The six zircon size fractions from the Muang Pan Gneissanalysed by conventional U/Pbtechniques (Table 1) 


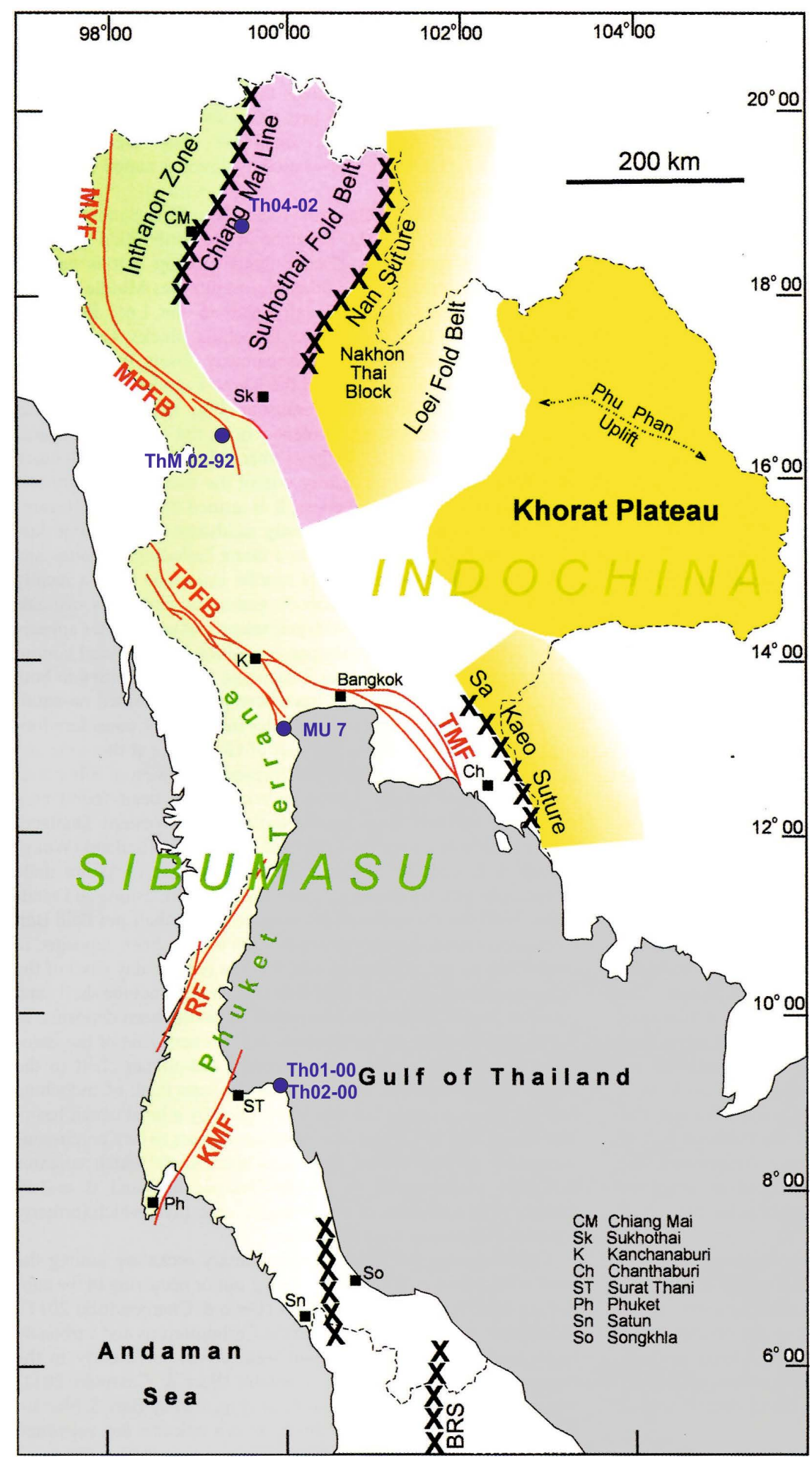

Figure 1. Sketch map of Thailand showing sample localities and structural elements of Thailand, including the two main terrains Sibumasu in the west and Indochina in the east as well as the Sukhothai Fold Belt in between outcropping crystalline basement rocks are marked in orange. MYF: Mae Yuam Fault; MPFB: Mae Ping Fault Belt; TPFB: Three Pagodas Fault Belt; TMF: Tha Mai Fault; RF: Ranong Fault; KMF: Khlong Marui Fault; BRS: Bentong-Raub Suture (modified after [42]). 
Table 1. U-Pb isotope data of zircon fractions from sample Th 04-02 obtained by conventional U-Pb technique.

\begin{tabular}{ccccccccccccc}
\hline \multicolumn{3}{c}{ Concentrations } & \multicolumn{4}{c}{ Measured Ratios } & \multicolumn{3}{c}{ Calculated Ratios } & \multicolumn{3}{c}{ Apparent Ages in Ma } \\
\hline $\mathrm{U}$ ppm & Pb ges. & Pb. rad & $\mathrm{Pbcom}$ & ${ }^{206} \mathrm{~Pb} /{ }^{204} \mathrm{~Pb}$ & ${ }^{207} \mathrm{~Pb} /{ }^{206} \mathrm{~Pb}$ & ${ }^{208} \mathrm{~Pb} /{ }^{206} \mathrm{~Pb}$ & ${ }^{206} \mathrm{~Pb} /{ }^{238} \mathrm{U}$ & ${ }^{207} \mathrm{~Pb} /{ }^{235} \mathrm{U}$ & ${ }^{207} \mathrm{~Pb} /{ }^{206} \mathrm{~Pb}$ & ${ }^{206} \mathrm{~Pb} /{ }^{238} \mathrm{U}$ & ${ }^{207} \mathrm{~Pb} /{ }^{235} \mathrm{U}$ & ${ }^{207} \mathrm{~Pb} /{ }^{206} \mathrm{~Pb}$ \\
256 & 21.6 & 21.6 & 0 & 5051 & 0.077404 & 0.075546 & 0.085929 & 0.884963 & 0.074694 & 531 & 644 & 1060 \\
246 & 23.2 & 23.2 & 0.06 & 7668 & 0.085612 & 0.073304 & 0.094876 & 1.097510 & 0.083897 & 584 & 752 & 1290 \\
330 & 29.3 & 29.2 & 0.11 & 6609 & 0.076191 & 0.098515 & 0.088057 & 0.900312 & 0.074153 & 544 & 652 & 1046 \\
506 & 41.6 & 41.6 & 0.02 & 9882 & 0.076612 & 0.067118 & 0.084049 & 0.872321 & 0.075274 & 520 & 637 & 1076 \\
1051 & 83.8 & 83.3 & 0.48 & 5522 & 0.073429 & 0.059606 & 0.081942 & 0.801749 & 0.070963 & 508 & 598 & 956 \\
2587 & 214.0 & 187.9 & 26.14 & 428 & 0.098646 & 0.124873 & 0.075973 & 0.689793 & 0.065850 & 472 & 533 & 802 \\
\hline
\end{tabular}

scatter around a reference discordia with a lower intercept at $428 \pm 19$ Ma and pointto an upper intercept at some 2190 Ma (Figure 2(a)). The pattern of the analysed fractions shows a normal distribution with respect to Uranium content and grain size ([19]). The scattering of the data points is most probably caused by loss of $\mathrm{Pb}$ in the slightly alterated outer rims of the zircons as to be seen in Figure 3. The composition of the zircon grains is quite simple consisting of a detrital rounded core and well developed zoned overgrowth. Therefore the discordia defines a mixing line between an inherited age of provenance and a new growth during high-grade metamorphism. To our opinion the lower intercept reflects the minimum age of the last high-grade metamorphism, whereas the upper intercept is interpreted to be the age of the source area of the protolith. The mean crustal residence time of the sample Th04-02 is determined by Sm/Nd WR analysis (Table 2) yielding a model age of $1836 \mathrm{Ma}\left(\mathrm{T}_{\mathrm{DM}}\right)$, which is a widespread modelage for the gneisses in N-Thailand ([15]). This model age determines the source area of the protoliths for the gneiss to be Paleoproterozoic. The age information of provenance given by the upper intercept of the U/Pbdiscordia is slightly older for the history of the zircon minerals, also pointing to the Paleoproterozoic. The cooling history of the Muang Pan gneissis documented by Rb-Sr and K-Ar dating on micas presented in Table 2 and Table 3. Rb-Sr analyses on muscovite-WR yielded an age of $357 \pm 1$ Ma considered to reflect the $500^{\circ} \mathrm{C} \pm 50^{\circ} \mathrm{C}$ cooling ([20]). The Rb-Srbiotite-WR reference line led to an age of $346 \pm 0.2 \mathrm{Ma}$, which is interpreted as cooling below $300^{\circ} \mathrm{C} \pm 50^{\circ} \mathrm{C}$ ([20]). K-Ar datingon muscovite yielded $352.2 \pm 7 \mathrm{Ma}$ and $352.0 \pm 7 \mathrm{Ma}$ for biotite, respectively. These ages are considered to document the cooling below $425^{\circ} \mathrm{C}$ for muscovite ([21]) and $300^{\circ} \mathrm{C} \pm 50^{\circ} \mathrm{C}$ for biotite [22], respectively. In sum, all mica data indicate rapid cooling in the Early Carboniferous time, around 357 to 346 Ma. This cooling is most probably related to tectonic activities like uplift and exhumation.

These results encouraged us to investigate the zircons of the Muang Pan Gneiss by SHRIMP analyses (Table 4). The results of the analysed spots are shown in Figure 2(b). The cathodoluminescence images of the analysed zircons from sample Th 04-02 including measurement spots, ages and calculated errors are displayed in Figure 3. Fourteen of the 22 analysed spots give a slightly older age of $445.2 \pm 3.8 \mathrm{Ma}$ which corresponds within limits of error to the conventional multigrain zircon age of $428 \pm 19$ Ma. This is strong evidence that a Middle Ordovician high-grade metamorphic event provoked the new growth of the zircons. The slightly younger ages obtained on some spots at the rims (spot 14 and 16) are related to lead loss by alteration whereas older ages from the cores (spot 2, 5, 15, 18 and 20) are interpreted to represent mixing ages of detrital zircons partly resetted by new overgrowth during Ordovician times.

\subsection{ThM 02-92. Paragneiss, Khlong Lan National Park, NW-Thailand ( $\left.16^{\circ} 12^{\prime} \mathrm{N} / 99^{\circ} 16^{\prime} \mathrm{E}\right)$}

The Khlong Lanparagneisscrops out in the Khlong Lan National Park, southern continuation of the Bhumibol and Lan Sang basement complexes. The rock sequence is composed of gneiss, schist, calc-silicate and marble. The gneiss is considered to be part of a paragneiss sequence which comprises quartz, feldspar and biotite covering the major part of the area. Ductile and brittle deformation features can be observed in the gneissic layering and are more pronounced towards the east ([23]).

Sample ThM 02-92, (Figure 1) represents a light grey altered augengneiss which was dated by conventional multigrain technique by [15]. The results showed the expected Indosinian age with a slight younging (174 \pm 5 Ma) and a further HT metamorphic overprint in the Lower Cretaceous (119 -10/+8 Ma). Similar young over 


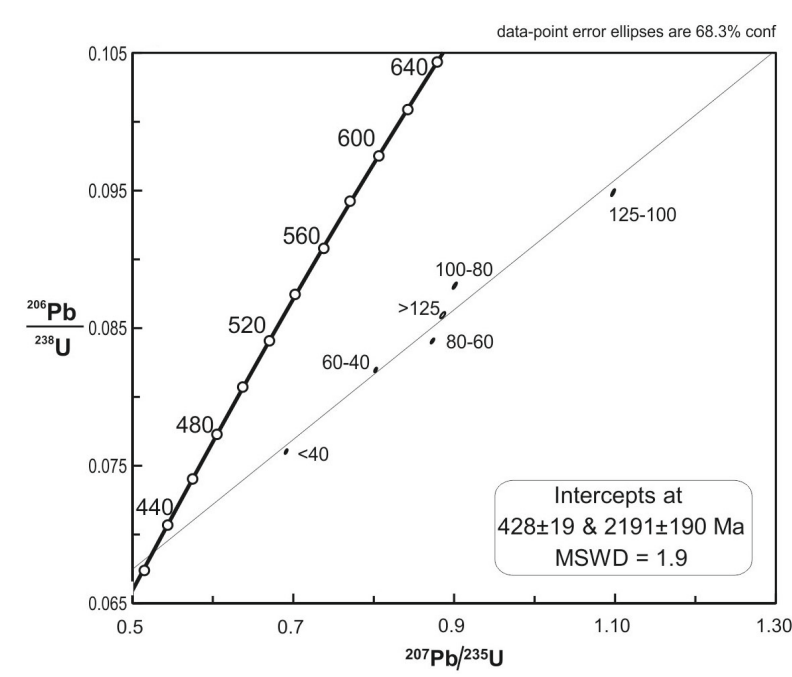

(a) Th 04-02

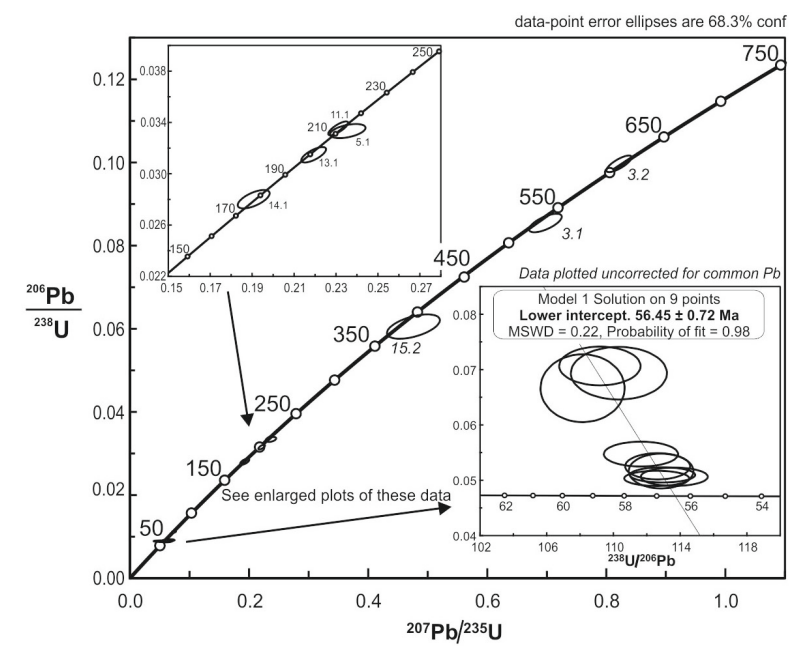

(c) Th 01-00

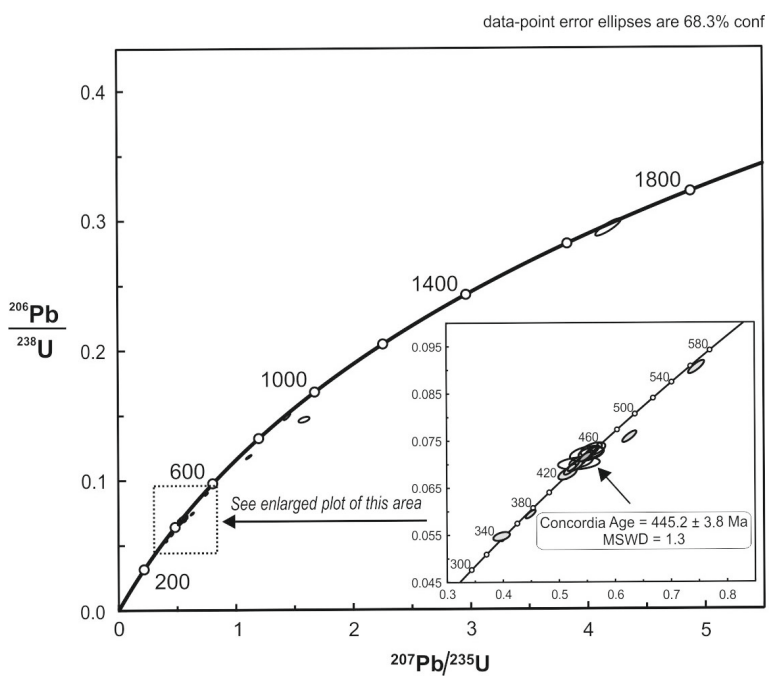

(b) Th 04-02

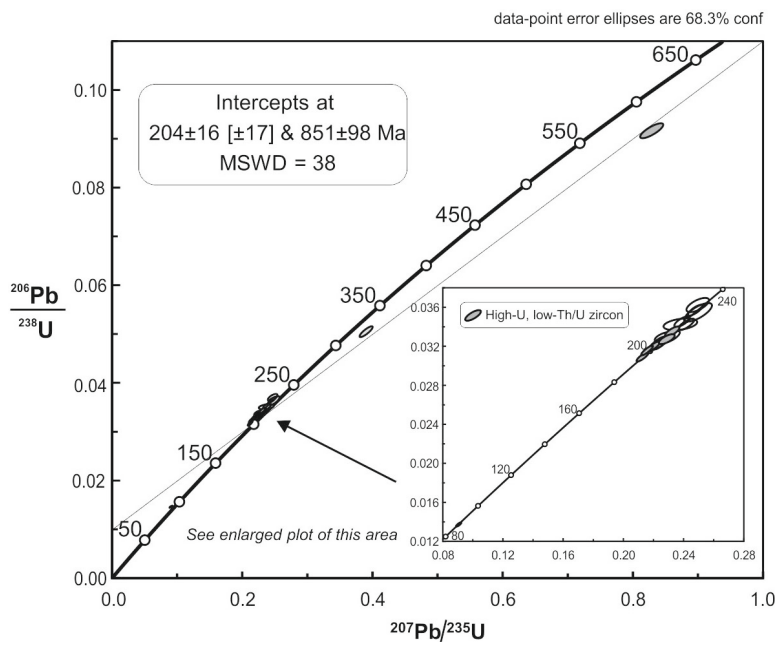

(d) ThM 02-92

Figure 2. Concordia plots of samples dated by conventional U-Pb technique. Plots are created using the program of [36].

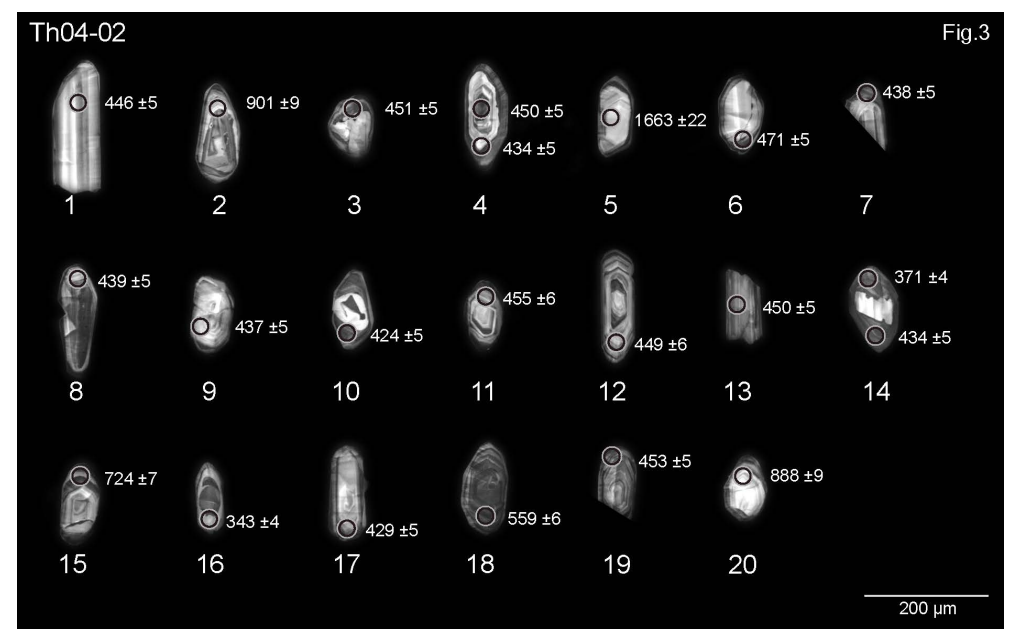

Figure 3. Cathodoluminescence images of the analysed zircons from sample Th 04-02 including measurement spots, ages and calculated errors. 
Table 2. Sm/Nd-Rb/Sr data from sample Th 04-02.

\begin{tabular}{|c|c|c|c|c|c|c|c|c|c|c|c|c|}
\hline \multicolumn{7}{|c|}{ Concentrations [ppm] } & \multicolumn{2}{|l|}{ Ratios } & \multirow[b]{2}{*}{${ }^{143} \mathrm{Nd} /{ }^{144} \mathrm{Nd}$} & \multirow[b]{2}{*}{$2 \sigma$ error } & \multirow[b]{2}{*}{$\mathrm{T}_{\mathrm{DM}}^{\mathrm{Nd}} * \mathrm{Ma}$} & \multirow[b]{2}{*}{$\varepsilon_{\mathrm{CHUR}}$} \\
\hline Type of sample & $\mathrm{Sm}$ & $\mathrm{Nd}$ & $\mathrm{Rb}$ & $\mathrm{Sr}$ & ${ }^{87} \mathrm{Rb} /{ }^{86} \mathrm{Sr}$ & ${ }^{87} \mathrm{Sr} /{ }^{86} \mathrm{Sr}$ & $2 \sigma$ error & ${ }^{147} \mathrm{Sm} /$ & & & & \\
\hline Whole rock & 5.72 & 30.53 & 121 & 139 & 2.52 & 0.749267 & 0.000019 & 0.11328 & 0.511935 & 0.000005 & 1836 & -13.72 \\
\hline Biotite & & & 497 & 8.94 & 174.77 & 1.597561 & 0.000381 & & & & & \\
\hline Muscovite & & & 260 & 51.7 & 14.688 & 0.811037 & 0.000222 & & & & & \\
\hline
\end{tabular}

${ }^{*} \mathrm{~T}_{\mathrm{DM}}^{\mathrm{Nd}}$ was calculated according to the model of [40]. The analytical procedure and data reductions are described in [41].

Table 3. K/Ar analytical data from sample Th 04-02.

\begin{tabular}{cccccc}
\hline Sample No. Th 04-02. & $\mathrm{K}_{2} \mathrm{O}(\mathrm{Wt} . \%)$ & ${ }^{40} \mathrm{Ar}^{*}(\mathrm{nl} / \mathrm{g}) \mathrm{STP}$ & ${ }^{40} \mathrm{Ar}^{*}(\%)$ & Age (Ma) & $2 \sigma$-Error (Ma) \\
\hline Muscowite & 10.70 & 134.21 & 97.75 & 352.2 & 7.2 \\
Biotite & 8.49 & 106.44 & 96.98 & 352.0 & 7.7 \\
\hline
\end{tabular}

The analytical procedure is given in [43].

prints with Upper Cretaceous ages are reported from the orthogneisses of the DoiInthanon (84 \pm 2 Ma, $72 \pm 1$ Ma, [24]) and a discordant dyke within the Larn Sang Gneiss (concordant monazite of $76 \pm 1 \mathrm{Ma}$, [15]). These overprints were mainly of local and thermal character and caused a lead loss in the zircons, as discussed in [1]. The SHRIMP analyses on zircons from ThM 02-92 (Figure 2(d) and Figure 4) were undertaken to verify this theory. Eighteen out of twenty-one spots confirm the Indosinian age for the formation of the oscillatory zirconrims with an average age of $204 \pm 17$ Ma. Only zircon 16 reflects an old core with an age of $560 \pm 6 \mathrm{Ma}$ as well as an altered rim with an Upper Cretaceous age of $88 \pm 1$ Ma. Again the SHRIMP ages confirm the interpretation of the conventional $\mathrm{U} / \mathrm{Pb}$ data for an Indosinian formation age of the Khlong Lhan Gneiss with a weakly superimposed thermal overprint during Cretaceous time as stated by [1].

\subsection{Th 01-00. Amphibolebearing Gneiss from the Khao Phra Mountain, S-Thailand} $\left(09^{\circ} 07^{\prime} \mathrm{N}-99^{\circ} 52^{\prime} \mathrm{E}\right)$

The high-grade metamorphic rocks of gneiss and schist in the lower southern peninsula occur only along the east coast at Khanom and Sichon districts, Nakhon Si Thammarat near Surat Thani province. The complexes were recognized and divided by [25] into two sub-units, from lower to upper units including, the Nai Plao Beach Gneiss and the Khao Yoi Schist. In the lower unit biotite gneiss with coarse grained porphyroblastic and equigranular textures are intercalated with sillimanite gneiss. Both, orthoclase and microcline are common porphyroblasts and usually engulf biotite, quartz and the needle-like or fibrolite sillimanite. Garnet was sometimes found as smaller sizedporphyroblast. In places, when cataclastically deformed, these both feldspars and garnet porphyroblastsmay show the same sense of rotational movement. The upper unit consists of schist, quartzite and small lenses of calc-silicate and marble.

Khao Phra amphibole bearing gneiss in this paper is considered to be the lower unit of the above mentioned gneissic complex. The contact between these high-grade metamorphic rocks and the adjacent Ordovician Thungsong limestone and Upper Paleozoic rocks are obscured by thick overburden and dense vegetation. However, all the Paleozoic rock formations and the basement rocks share similar tectonic foliation and alignments.

Previous $\mathrm{U} / \mathrm{Pb}$ dating on zircons of a amphibolebearing gneiss (Th 01-00, Th 02-00) from the Khao Phra Mountain (Figure 1) showed a very young U/Pb lower intercept of Eocene age (45+12/-18 Ma) as well as an upper intercept age of $452+93 /-84 \mathrm{Ma}$ ([1]), the latter similar to the age obtained on sample Th 04-02 from the north (s.a.). The lower intercept age determined by conventional U/Pb techniques was corroborated by the $\mathrm{K} / \mathrm{Ar}$ data from a micaceous gneiss (Th 5-00) sampled $\sim 25 \mathrm{~km}$ west of the above-mentioned amphibole-bearing gneiss which showed a muscovite cooling age of $43 \pm 4$ Ma reflecting uplift and cooling in the Eocene ([1]). In order to verify these ages additional SHRIMP analyses were performed. The data are shown in Table 4 and the cathodoluminescence images of the analysed zircons from sample Th 01-00 including measurement spots, ages and calculated errors are shown in Figure 5. 
Table 4. Summary of SHRIMP U-Th-Pb zircon data.

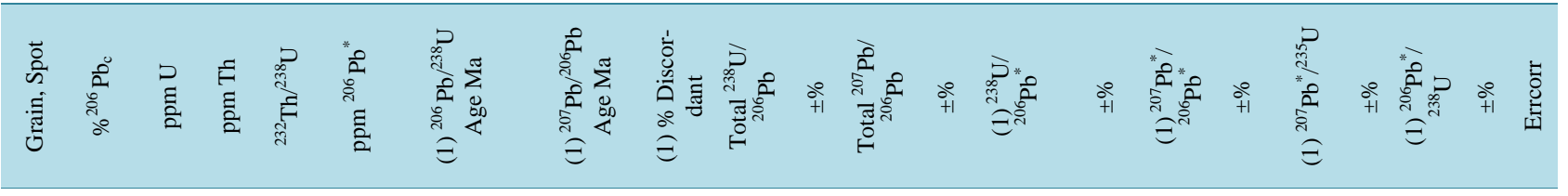

Th 1-00 (Paleogenezircons only)

$\begin{array}{llllllll}1.1 & 0.95 & 539 & 798 & 1.53 & 4.2 & 56.9 & \pm 0.75\end{array}$

$\begin{array}{llllllll}2.1 & 0.40 & 1256 & 922 & 0.76 & 9.6 & 56.8 & \pm 0.65\end{array}$

$\begin{array}{lllllllll}4.1 & 0.48 & 1361 & 64 & 0.05 & 10.4 & 56.6 & \pm 0.65\end{array}$

$\begin{array}{llllllll}6.1 & 2.97 & 308 & 40 & 0.13 & 2.4 & 57.1 & \pm 0.86\end{array}$

$\begin{array}{lllllllll}7.1 & 0.57 & 1352 & 1795 & 1.37 & 10.3 & 56.6 & \pm 0.66\end{array}$

$\begin{array}{llllllll}8.1 & 2.80 & 264 & 461 & 1.80 & 2.1 & 56.6 & \pm 10\end{array}$

$\begin{array}{llllllll}9.1 & 2.46 & 239 & 174 & 0.75 & 1.9 & 57.9 & \pm 0.94\end{array}$

$\begin{array}{llllllll}10.1 & 0.44 & 1157 & 1096 & 0.98 & 8.7 & 56.2 & \pm 0.66\end{array}$

$\begin{array}{llllllll}12.1 & 0.68 & 1175 & 1182 & 1.04 & 9.0 & 56.59 & \pm 0.66\end{array}$ $\begin{array}{llllll}111.6 & 1.3 & 0.0547 & 2.7 & 0.00887 & 0.00012\end{array}$ $\begin{array}{llllll}112.6 & 1.1 & 0.0503 & 1.8 & 0.00885 & 0.00010\end{array}$ $\begin{array}{llllll}112.9 & 1.1 & 0.05095 & 1.8 & 0.00881 & 0.00010\end{array}$ $\begin{array}{llllll}109.2 & 1.5 & 0.0707 & 3.3 & 0.00889 & 0.00013\end{array}$ $\begin{array}{lllllll}112.8 & 1.1 & 0.0517 & 4 & 0.00881 & 0.00010\end{array}$ $\begin{array}{lllllll}110.3 & 1.7 & 0.0694 & 4.5 & 0.00881 & 0.00016\end{array}$ $\begin{array}{llllll}108.1 & 1.5 & 0.0666 & 6.1 & 0.00902 & 0.00015\end{array}$ $\begin{array}{llllll}113.6 & 1.2 & 0.0506 & 2.2 & 0.00876 & 0.00010\end{array}$ $\begin{array}{llllll}112.6 & 1.1 & 0.0525 & 3 & 0.00882 & 0.00010\end{array}$ Th 1-00 (older "Inherited" grains)

$\begin{array}{lllllllllllllllll}3.1 & 0.20 & 870 & 686 & 0.81 & 63.9 & 528.50 & \pm 8.9 & 567 & \pm 41 & 6.79 & 11.70 & 1.80 & 0.06 & 1.80 & 11.70 & 1.80\end{array}$

$\begin{array}{lllllllllllllllll}3.2 & 0.02 & 757 & 955 & 1.30 & 64.8 & 612.30 & \pm 7.8 & 589 & \pm 23 & -3.96 & 10.03 & 1.30 & 0.06 & 0.94 & 10.04 & 1.30\end{array}$

$\begin{array}{lllllllllllllllll}5.1 & 0.46 & 825 & 91 & 0.11 & 23.7 & 211.40 & \pm 2.3 & 252 & \pm 49 & 16.11 & 29.89 & 1.10 & 0.05 & 1.10 & 30 & 1.10\end{array}$

$\begin{array}{lllllllllllllllll}11.1 & 0.05 & 3043 & 138 & 0.05 & 87.8 & 212.70 & \pm 2.2 & 193 & \pm 19-10.21 & 29.79 & 1.10 & 0.05 & 0.56 & 29.82 & 1.10\end{array}$

$\begin{array}{lllllllllllllllll}13.1 & 0.20 & 1468 & 113 & 0.08 & 39.8 & 199.80 & \pm 2.5 & 219 & \pm 29 & 8.77 & 31.71 & 1.30 & 0.05 & 0.85 & 31.76 & 1.30\end{array}$

$\begin{array}{lllllllllllllllll}14.1 & 0.15 & 1473 & 174 & 0.12 & 35.5 & 178.10 & \pm 3 & 166 & \pm 48 & -7.29 & 35.64 & 1.70 & 0.05 & 1.70 & 35.70 & 1.70\end{array}$

$\begin{array}{lllllllllllllllll}15.1 & 0.15 & 2659 & 423 & 0.16 & 25.5 & 71.34 & \pm 0.77 & 66 & \pm 66 & -8.09 & 89.72 & 1.10 & 0.05 & 1.20 & 89.86 & 1.10\end{array}$ Th4-02

$\begin{array}{lllllllllll}1.1 & 0.00 & 539 & 315 & 0.60 & 33.2 & 445.80 & \pm 4.7 & 450 & \pm 21 & 0.93\end{array}$

$\begin{array}{llllllllllllllll}2.1 & 0.06 & 493 & 221 & 0.46 & 63.6 & 901 & \pm 9.1 & 912 & +14 & 1.21 & 6.66 & 1.10 & 0.07 & 0.61 & 6.67\end{array}$

$\begin{array}{lllllllllllllllll}3.1 & 0.11 & 1100 & 151 & 0.14 & 68.6 & 451.20 & \pm 4.6 & 448 & \pm 16 & -0.71 & 13.78 & 1.10 & 0.06 & 0.66 & 13.79 & 1.10\end{array}$

$\begin{array}{lllllllllllllllll}4.1 & 0.00 & 1091 & 13 & 0.01 & 65.2 & 433.60 & \pm 4.5 & 416 & \pm 16 & -4.23 & 14.37 & 1.10 & 0.06 & 0.72 & 14.37 & 1.10\end{array}$

$\begin{array}{lllllllllllllllll}4.2 & 0.07 & 1001 & 892 & 0.92 & 62.2 & 449.70 & \pm 5.3 & 458 & \pm 18 & 1.81 & 13.83 & 1.20 & 0.06 & 0.74 & 13.84 & 1.20\end{array}$

$\begin{array}{lllllllllllllllll}5.1 & 0.16 & 232 & 44 & 0.19 & 58.8 & 1663 & \pm 22 & 1679 & \pm 15 & 0.95 & 3.39 & 1.50 & 0.10 & 0.73 & 3.40 & 1.50\end{array}$

$\begin{array}{lllllllllllllllll}6.1 & 0.04 & 1189 & 60 & 0.05 & 77.4 & 471 & \pm 4.8 & 605 & \pm 16 & 22.15 & 13.19 & 1.10 & 0.06 & 0.67 & 13.19 & 1.10\end{array}$

$\begin{array}{lllllllllllllllll}7.1 & 0.12 & 1015 & 13 & 0.01 & 61.3 & 437.60 & \pm 4.6 & 438 & \pm 22 & 0.09 & 14.22 & 1.10 & 0.06 & 0.78 & 14.24 & 1.10\end{array}$

$\begin{array}{lllllllllllllllll}8.1 & 0.21 & 457 & 38 & 0.09 & 27.7 & 438.70 & \pm 4.9 & 446 & \pm 50 & 1.64 & 14.17 & 1.20 & 0.06 & 1.20 & 14.20 & 1.20\end{array}$

$\begin{array}{lllllllllllllllll}9.1 & 0.62 & 283 & 75 & 0.27 & 17.2 & 436.80 & \pm 5.3 & 440 & \pm 97 & 0.73 & 14.17 & 1.20 & 0.06 & 2.50 & 14.26 & 1.30\end{array}$

$\begin{array}{lllllllllllllllll}10.1 & 0.17 & 945 & 14 & 0.02 & 55.2 & 423.50 & \pm 5.1 & 429 & \pm 36 & 1.28 & 14.70 & 1.20 & 0.06 & 1.10 & 14.73 & 1.30\end{array}$

$\begin{array}{lllllllllllllllll}11.1 & 0.25 & 623 & 150 & 0.25 & 39.2 & 454.80 & \pm 5.8 & 449 & \pm 48 & -1.29 & 13.65 & 1.30 & 0.06 & 1.60 & 13.68 & 1.30\end{array}$

$\begin{array}{lllllllllllllllll}12.1 & 0.36 & 648 & 114 & 0.18 & 40.3 & 449.10 & \pm 6.4 & 435 & \pm 72 & -3.24 & 13.81 & 1.50 & 0.06 & 2.20 & 13.86 & 1.50\end{array}$

$\begin{array}{lllllllllllllllll}13.1 & 0.32 & 575 & 279 & 0.50 & 35.9 & 450.10 & \pm 4.9 & 456 & \pm 50 & 1.29 & 13.78 & 1.10 & 0.06 & 1 & 13.83 & 1.10\end{array}$

$\begin{array}{lllllllllllllllll}14.1 & 0.11 & 1046 & 12 & 0.01 & 62.7 & 434.10 & \pm 4.5 & 435 & \pm 23 & 0.21 & 14.34 & 1.10 & 0.06 & 0.73 & 14.35 & 1.10\end{array}$

$\begin{array}{lllllllllllllllll}14.2 & 0.04 & 1041 & 8 & 0.01 & 53.0 & 371.40 & \pm 3.8 & 428 & \pm 18 & 13.22 & 16.85 & 1.10 & 0.06 & 0.76 & 16.86 & 1.10\end{array}$

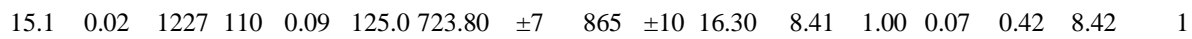

$\begin{array}{lllllllllllllllll}16.1 & 0.17 & 367 & 17 & 0.05 & 17.3 & 342.90 & \pm 3.7 & 345 & \pm 48 & 0.61 & 18.27 & 1.10 & 0.05 & 1.20 & 18.30 & 1.10\end{array}$

$\begin{array}{lllllllllllllllll}17.1 & 0.00 & 605 & 57 & 0.10 & 35.8 & 429 & \pm 4.5 & 416 & \pm 19 & -3.13 & 14.53 & 1.10 & 0.06 & 0.85 & 14.53 & 1.10\end{array}$

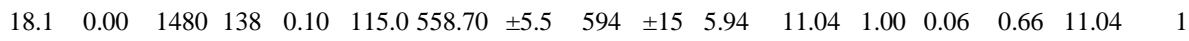

$\begin{array}{lllllll}0.06 & 1.90 & 0.70 & 2.60 & 0.09 & 1.8 & 0.68\end{array}$ $\begin{array}{lllllll}0.06 & 1.10 & 0.82 & 1.70 & 0.10 & 1.3 & 0.78\end{array}$ $\begin{array}{lllllll}0.05 & 2.10 & 0.24 & 2.40 & 0.03 & 1.1 & 0.46\end{array}$ $\begin{array}{llllllll}0.05 & 0.83 & 0.23 & 1.40 & 0.03 & 1.1 & 0.79\end{array}$ $\begin{array}{lllllll}0.05 & 1.30 & 0.22 & 1.80 & 0.03 & 1.3 & 0.71\end{array}$ $\begin{array}{lllllll}0.05 & 2.00 & 0.19 & 2.60 & 0.03 & 1.7 & 0.64\end{array}$ $\begin{array}{llllllll}0.05 & 2.80 & 0.07 & 3 & 0.01 & 1.1 & 0.36\end{array}$

$\begin{array}{lllllll}0.06 & 0.95 & 0.55 & 1.40 & 0.07 & 1.1 & 0.76\end{array}$ $\begin{array}{lllllll}0.07 & 0.67 & 1.44 & 1.30 & 0.15 & 1.1 & 0.85\end{array}$ $\begin{array}{lllllll}0.06 & 0.74 & 0.56 & 1.30 & 0.07 & 1.1 & 0.82\end{array}$ $\begin{array}{llllllll}0.06 & 0.72 & 0.53 & 1.30 & 0.07 & 1.1 & 0.83\end{array}$ $\begin{array}{llllllll}0.06 & 0.82 & 0.56 & 1.50 & 0.07 & 1.2 & 0.83\end{array}$ $\begin{array}{lllllll}0.10 & 0.83 & 4.18 & 1.70 & 0.29 & 1.5 & 0.87\end{array}$ $\begin{array}{lllllll}0.06 & 0.73 & 0.63 & 1.30 & 0.08 & 1.1 & 0.82\end{array}$ $\begin{array}{llllllll}0.06 & 1.00 & 0.54 & 1.50 & 0.07 & 1.1 & 0.73\end{array}$ $\begin{array}{lllllll}0.06 & 2.20 & 0.54 & 2.50 & 0.07 & 1.2 & 0.46\end{array}$

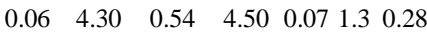
$\begin{array}{lllllll}0.06 & 1.60 & 0.52 & 2.10 & 0.07 & 1.3 & 0.61\end{array}$ $\begin{array}{lllllll}0.06 & 2.10 & 0.56 & 2.50 & 0.07 & 1.3 & 0.53\end{array}$ $\begin{array}{lllllll}0.06 & 3.20 & 0.55 & 3.60 & 0.07 & 1.5 & 0.41\end{array}$ $\begin{array}{lllllll}0.06 & 2.30 & 0.56 & 2.50 & 0.07 & 1.1 & 0.44\end{array}$ $\begin{array}{lllllll}0.06 & 1.10 & 0.53 & 1.50 & 0.07 & 1.1 & 0.71\end{array}$ $\begin{array}{llllllll}0.06 & 0.79 & 0.45 & 1.30 & 0.06 & 1.1 & 0.80\end{array}$ $\begin{array}{lllllll}0.07 & 0.48 & 1.11 & 1.10 & 0.12 & 1 & 0.91\end{array}$ $\begin{array}{lllllll}0.05 & 2.10 & 0.40 & 2.40 & 0.05 & 1.1 & 0.47\end{array}$ $\begin{array}{lllllll}0.06 & 0.85 & 0.52 & 1.40 & 0.07 & 1.1 & 0.79\end{array}$ $\begin{array}{lllllll}0.06 & 0.68 & 0.75 & 1.20 & 0.09 & 1 & 0.84\end{array}$ 


\section{Continued}

\begin{tabular}{|c|c|c|c|c|c|c|c|c|c|c|c|c|c|c|c|c|c|c|c|c|c|c|}
\hline 19.1 & 0.06 & 844 & 269 & 0.33 & 52.8 & 452.60 & \pm 4.6 & 455 & \pm 19 & 0.53 & 13.74 & 1.10 & 0.06 & 0.70 & 13.75 & 1.10 & 0.06 & 0.87 & 0.56 & 1.40 & 0.071 .1 & 0.77 \\
\hline 20.10 & 0.04 & 380 & 211 & 0.57 & 48.2 & .10 & \pm 8.9 & 1139 & \pm 33 & 22.03 & 6.77 & 1.10 & 0.08 & 1.60 & 77 & 0 & .08 & 1.60 & 1.58 & 2 & 0.151 .1 & 0.55 \\
\hline \multicolumn{23}{|c|}{ Th M2/92 } \\
\hline 1.1 & 0.15 & 513 & 407 & 0.28 & 47.1 & 229 & \pm 3 & 180 & $\pm 35-$ & -27.22 & 27.61 & 1.30 & 0.05 & 0.81 & 27.65 & 1.30 & 0.05 & 1.50 & 0.25 & 2 & 0.041 .3 & 0.66 \\
\hline 2.1 & 0.14 & 945 & 221 & 0.12 & 55.1 & 208.90 & \pm 2.4 & 227 & \pm 26 & 7.97 & 30.32 & 1.20 & 0.05 & 0.92 & 30.36 & 1.20 & 0.05 & 1.10 & 0.23 & 1.60 & 0.031 .2 & 0.72 \\
\hline 3.1 & 0.05 & 3792 & 36 & 0.01 & 105.0 & 204 & \pm 2.9 & 186 & \pm 21 & -9.68 & 31.08 & 1.40 & 0.05 & 0.83 & 31.10 & 1.40 & 0.05 & 0.89 & 0.22 & 1.70 & 0.031 .4 & 0.85 \\
\hline 4.1 & 0.10 & 821 & 41 & 0.01 & 106.0 & 204 & \pm 2.1 & 215 & \pm 16 & 5.12 & 31.08 & 1 & 0.05 & 0.54 & 31.11 & 1.00 & 0.05 & 0.68 & 0.22 & 1.20 & $0.03 \quad 1$ & 0.84 \\
\hline 5.1 & 0.06 & 390 & 88 & 0.03 & 93.1 & 202.60 & \pm 2.1 & 185 & \pm 17 & -9.51 & 31.30 & 1 & 0.05 & 0.56 & 31.32 & 1 & 0.05 & 0.72 & 0.22 & 1.30 & $0.03 \quad 1$ & 0.82 \\
\hline 6.1 & 0.14 & 484 & 307 & 0.21 & 42.0 & 208.60 & \pm 2.2 & 186 & \pm 36 & -12 . & 30.37 & 1.10 & 0.05 & 1.40 & 30.41 & 1.10 & 0.05 & 1.60 & 0.23 & 1.90 & 0.031 .1 & 0.56 \\
\hline 7.1 & 0.11 & 3511 & 115 & 0.03 & 101.0 & 212 & \pm 2.1 & 205 & \pm 20 & -3.41 & 29.87 & 1 & 0.05 & 0.58 & 29.91 & 1 & 0.05 & 0.87 & 0.23 & 1.40 & 0.031 & 0.76 \\
\hline 8.1 & 0.09 & 2502 & 175 & 0.07 & 69.8 & 205.90 & \pm 2.1 & 188 & \pm 20 & -9.52 & 30.78 & 1 & 0.05 & 0.70 & 30.81 & 1 & 0.05 & 0.86 & 0.22 & 1.30 & 0.031 & 0.77 \\
\hline 10.1 & 0.00 & 214 & 120 & 0.03 & 114 & 20 & \pm & 168 & \pm 14 & -19 & 31.71 & 1 & 0.05 & 0.58 & 31 & 1 & 0.05 & .58 & 0.21 & 1.20 & $0.03 \quad 1$ & 0.87 \\
\hline 10.2 & 0.04 & 1615 & 781 & 0.50 & 47.5 & 216.70 & \pm 2.4 & 241 & \pm 19 & 10.08 & 29.24 & 1.10 & 0.05 & 0.80 & 29.25 & 1.10 & 0.05 & 0.84 & 0.24 & 1.40 & 0.031 .1 & 0.80 \\
\hline 11.1 & 0.11 & 1694 & 801 & 0.49 & 50.2 & 218.30 & \pm 2.2 & 201 & \pm 22 & -8.61 & ר & 1 & 0.05 & 0.76 & 29.03 & 1 & 0.05 & 0.96 & 0.24 & 1.40 & 0.031 & 0.74 \\
\hline 12.1 & 0.00 & 2594 & 270 & 0.11 & $68 . .8$ & 196 & \pm 2 & 183 & \pm 15 & -7.10 & 32.39 & 1 & 0.05 & 0.65 & 32.39 & 1 & 0.05 & 0.65 & 0.21 & 1.20 & 0.031 & 0.85 \\
\hline 13.1 & 0.17 & 2849 & $\begin{array}{c}115 \\
1\end{array}$ & 0.42 & 80.1 & 207.30 & \pm 2.1 & 217 & \pm 24 & 4.47 & 30.55 & 1 & 0.05 & 0.77 & 30.60 & 1 & 0.05 & 1 & 0.23 & 1.50 & 0.031 & 0.70 \\
\hline 13.2 & 0.08 & 4213 & 135 & 0.03 & 117 & 205.40 & \pm 3.1 & 220 & \pm 14 & 6.64 & 30.86 & 1.50 & 0.05 & 0.50 & 30.88 & 1.50 & 0.05 & 0.61 & 0.23 & 1.70 & 0.031 .5 & 0.93 \\
\hline 14.1 & 0.05 & 791 & 254 & 0.33 & 33.7 & 312 & \pm 4.5 & 497 & \pm 20 & 37.22 & 20.15 & 1.50 & 0.06 & 0.87 & 20.16 & 1.50 & 0.06 & 0.90 & 0.39 & 1.70 & 0.051 .5 & 0.86 \\
\hline 15.1 & 0.50 & 445 & 357 & 0.83 & 13.1 & 216.70 & \pm 2.4 & 200 & \pm 68 & -8.35 & 29.10 & 1.10 & 0.05 & 1.40 & 29.24 & 1.10 & 0.05 & 2.90 & 0.24 & 3.10 & 0.031 .1 & 0.37 \\
\hline 16.1 & 0.00 & 5241 & 29 & 0.01 & 61.8 & 87.93 & \pm 0.9 & 07 & \pm 17 & 9.35 & 72.82 & 1 & 0.05 & 0.71 & 72.82 & 1 & 0.05 & 0.71 & 0.09 & 1.30 & $0.01 \quad 1$ & 0.82 \\
\hline 16.2 & 0.11 & 560 & 184 & 0.34 & 43.7 & 559.30 & \pm 5.9 & 806 & \pm 17 & 30.61 & 11.02 & 1.10 & 0.07 & 0.75 & 11.03 & 1.10 & 0.07 & 0.83 & 0.83 & 1.40 & 0.091 .1 & 0.80 \\
\hline 18.1 & 0.00 & 3851 & 48 & 0.01 & 106 & 203.90 & \pm 2.5 & 193 & \pm 20 & -5.65 & 31.12 & 1.30 & 0.05 & 0.87 & 31.12 & 1.30 & 0.05 & 0.87 & 0.22 & 1.50 & 0.031 .3 & 0.82 \\
\hline 15.2 & 0.89 & 176 & 57 & 0.33 & 9.2 & 379 & \pm 12 & $483=$ & \pm 120 & 21.53 & 16.43 & 3.20 & 0.06 & 1.90 & 16.52 & 3.20 & 0.06 & 5.30 & 0.47 & 6.20 & $0.06 \quad 3.2$ & 0.52 \\
\hline
\end{tabular}

Errors are 1-sigma; $\mathrm{Pbc}$ and $\mathrm{Pb}^{*}$ indicate the common and radiogenic portions, respectively, error in Standard calibration was $0.21 \%$ (not included in above errors but required when comparing data from different mounts), (1) No correction for common lead was aplied to data used for Concordia plot due to low concentration of radiogenic and high concentration of common lead. (2) Common Pb corrected using measured ${ }^{204} \mathrm{~Pb}$.

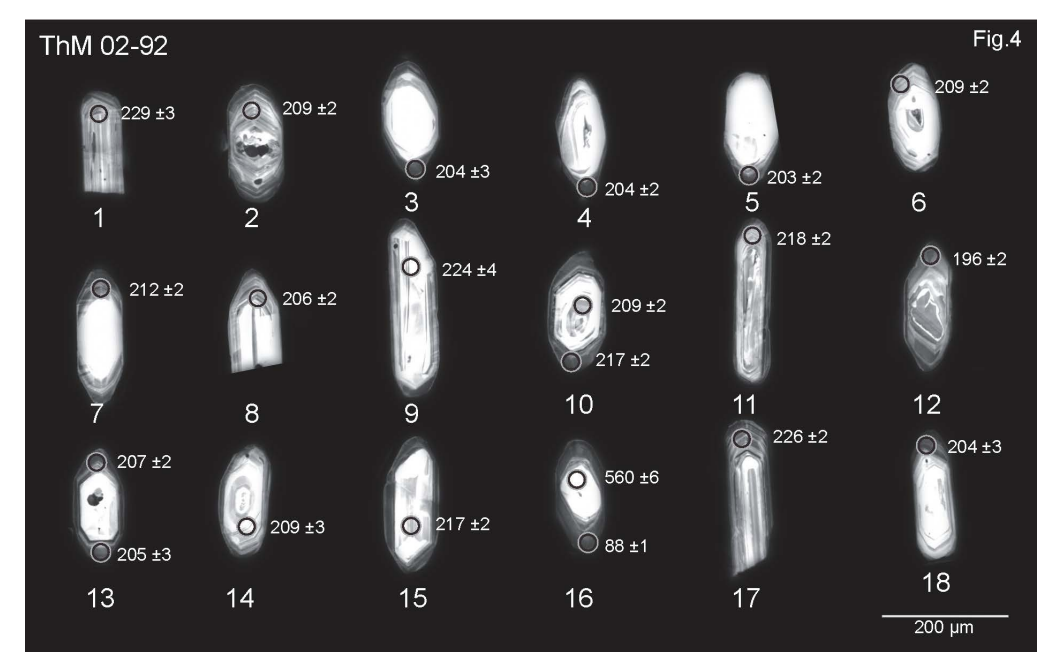

Figure 4. Cathodoluminescence images of the analysed zircons from sample ThM 02-92 including measurement spots, ages and calculated errors. 


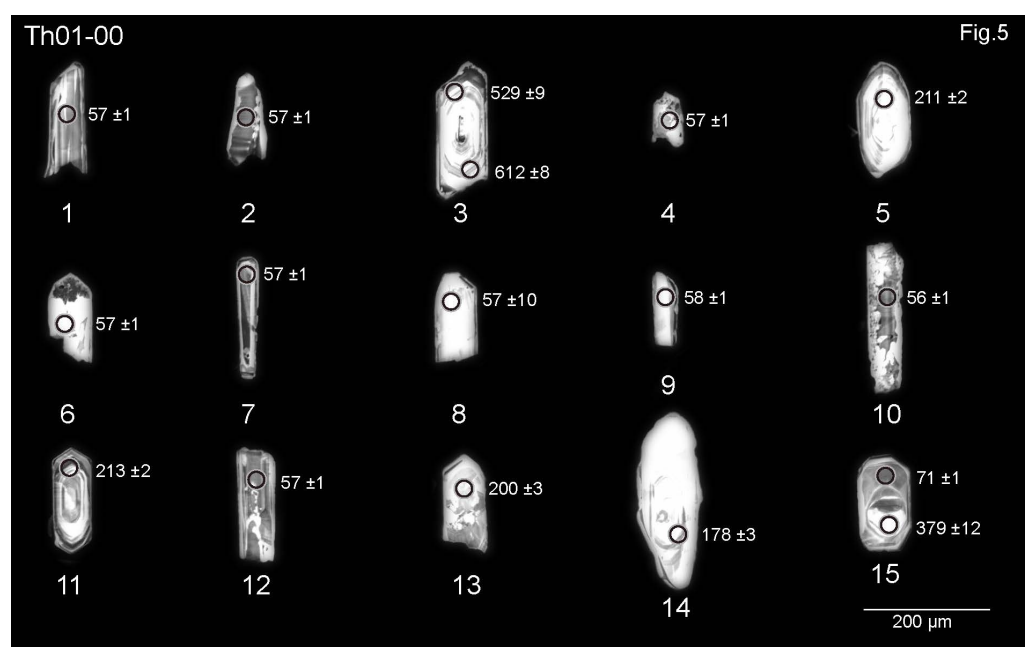

Figure 5. Cathodoluminescence images of the analyzed zircons from sample Th 01-00 including measurement spots, ages and calculated errors.

Nine SHRIMP spots within the fifteen analysed zircons confirm aslightly older Paleocene age of $56.45 \pm 0.72$ Ma (Figure 2(c)). Comparable Eocene ages were reported by [6] (U-Pb LA-ICP-MS on zircons and Rb-Sr on micas), these authors relate Middle Eocene data to dextral ductile deformation, and Late Eocene data to dextral transpressional deformation in the Khlong Marui shearzone. Our data originate from a location approximately $70 \mathrm{Km}$ SE of the shearzone.

The rest of our analyses from the cores of the zircons show a few concordant ages between 178 and 612 Ma. It is interesting to note that three spots $(5,11$ and 13$)$ reflect older Indosinianages for the inner parts of the zircons, The Ordovician age of 452 Ma obtained by convential multigrain dating ([1]) could not be confirmed. However, recently [26] reported an LA-ICP-MS U-Pb age of $477 \pm 7$ Ma for the Khao Dat Fa granite from the Khanom area of Peninsular Thailand. These authors interpreted this age as evidence that the Sibumasu Block has a crystalline basement that was formed during the Ordovician time. Contrastingly, [6] published a great number of far older ages up to Archean times for the inner parts of their zircons

\subsection{MU 7. Ban Khao Takiab Orthogneiss, Central Thailand ( $\left.12^{\circ} 54{ }^{\prime} \mathrm{N}-99^{\circ} 57^{\prime} \mathrm{E}\right)$}

The high-grade metamorphic rocks of Hua Hin-Pran Buri including Ban KhaoTakiab area, central Thailand were first described by and [27] [28]. The garnet-bearing biotiteorthogneiss extends approximately $60 \mathrm{~km} \mathrm{~N}-\mathrm{S}$ from the western part of Cha Am districtto Pran Buri district. The rocks are coarse to very coarse grained and porphyroblastic showing well defined myloniticandcataclastic overprints, most intensive along a major northeast-southwest strike slip fault zone which is considered to be a branch of the Three Pagodas Fault. The orthogneissis bordered by a succession of paragneiss and meta-sedimentary sequences from the lowermost horizon to the top horizon as follows: metapelite (mica sillimanite schist) and gneiss, calc-silicate, quartzofeldspathicrocks and marble, quartzite and marble ([28]). Sample MU 7 is a coarse grained porphyroblastic garnet-bearing biotite gneiss collected at Ban Khao Takiab showing cataclasticoverprint. It used to be considered as part of the Hua Hin-Pran Burimylonite zone. [29] reportedbiotite K/Ar cooling ages of 34 and $32 \mathrm{Ma}$ from this gneiss. A Rb/Srisochron from the intruding Hub Kapong foliated granite which is part of the Hub Kapong Batholith yielded an age of $210 \pm 4$ Ma ([27] [30]).

In order to verify the time of the main metamorphic event leading to the formation of the gneissic texture and the related garnets as well as to prove whether the garnets show a multiple growth age-zonation leach experiments were performed. The $\mathrm{Pb} / \mathrm{Pb}$ data obtained are presented in Table 5 and plotted in Figure 6. The data defines a reference line with a slope corresponding to an age of $224 \pm 120 \mathrm{Ma}$. Even if the fit (MSWD = 17) is not ideal, all data points lie within limits of error on the reference line and show no sign of older cores within the analyzed garnets. This fact is interpreted as a proof that the garnet were solely formed during the high-grade metamorphism which led to the formation of the gneiss during Indosinian times. The thermal pulse which resetted the $\mathrm{K} / \mathrm{Ar}$ isotope system in the biotites did not affect neither the $\mathrm{Pb} / \mathrm{Pb}$ nor the $\mathrm{Rb} / \mathrm{Sr}$ system. 
Table 5. $\mathrm{Pb} / \mathrm{Pb}$ data for the isotope ratios obtained by step-wise leaching on garnets from sample MU-7.

\begin{tabular}{cccccccccc}
\hline \multicolumn{7}{c}{ Pb isotope data of Pb leach experiment for orthogneiss MU-7 (125 - $250 \mu \mathrm{m}$ garnet fraction) } \\
\hline Step & Time & Temperature & Acid & ${ }^{206} \mathrm{~Pb} /{ }^{204} \mathrm{~Pb}$ & $2 \mathrm{se}$ & ${ }^{207} \mathrm{~Pb} /{ }^{204} \mathrm{~Pb}$ & 2se & ${ }^{208} \mathrm{~Pb} /{ }^{204} \mathrm{~Pb}$ & $2 \mathrm{se}$ \\
\hline 1 & $10 \mathrm{~min}$ & Cold & $0.5 \mathrm{~N} \mathrm{HBr}$ & 19.242 & 0.009 & 15.409 & 0.008 & 37.744 & 0.018 \\
2 & $30 \mathrm{~min}$ & $120^{\circ} \mathrm{C}$ & $1 \mathrm{~N} \mathrm{HBr}$ & 22.204 & 0.004 & 15.542 & 0.003 & 38.313 & 0.006 \\
3 & $2.5 \mathrm{~h}$ & $120^{\circ} \mathrm{C}$ & $4 \mathrm{~N} \mathrm{HBr}$ & 53.265 & 0.037 & 17.135 & 0.014 & 63.852 & 0.040 \\
4 & $4 \mathrm{~h}$ & $120^{\circ} \mathrm{C}$ & $8.8 \mathrm{~N} \mathrm{HBr}$ & 58.390 & 0.070 & 17.264 & 0.045 & 139.498 & 0.158 \\
5 & $18 \mathrm{~h}$ & $120^{\circ} \mathrm{C}$ & $8.8 \mathrm{~N} \mathrm{HBr}$ & 68.692 & 0.279 & 17.859 & 0.159 & 153.248 & 0.552 \\
\hline
\end{tabular}

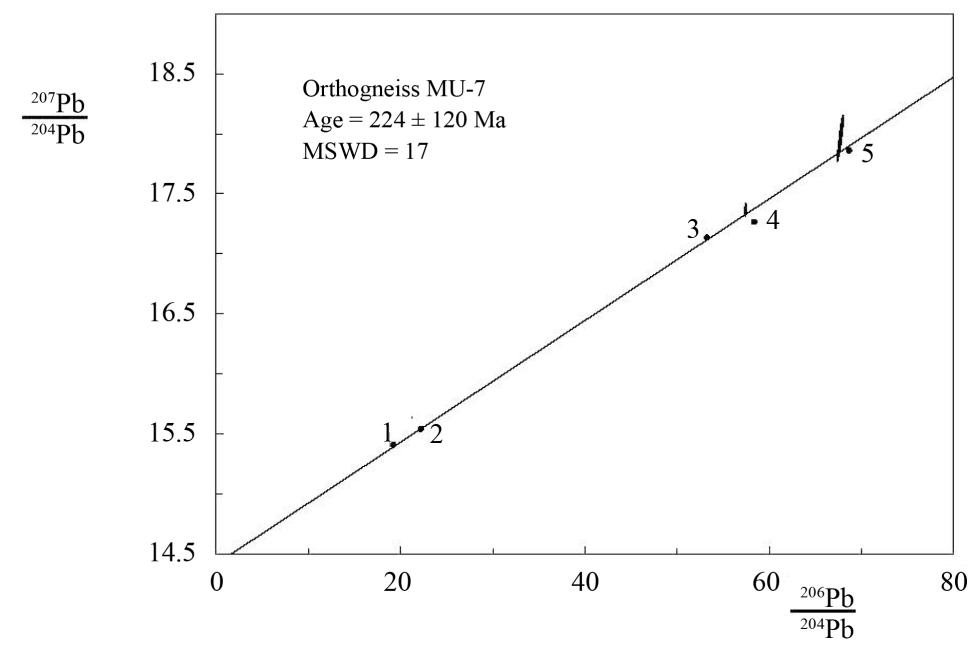

Figure 6. ${ }^{207} \mathrm{~Pb} /{ }^{204} \mathrm{~Pb}$ vs. ${ }^{206} \mathrm{~Pb} /{ }^{204} \mathrm{~Pb}$ plot for the isotope ratios obtained by step-wise leaching on garnets from sample MU-7.

\section{Conclusion}

The SHRIMP data as well as the PbSL age of syn-metamorphic garnets confirm the conclusions for the evolution of the basement rocks of Thailand as proposed by [1]. A Precambrian age for the high-grade metamorphic genesis of the crystalline rocks in Thailand can be excluded. A few locations show an early metamorphic event of Middle Ordovician age. Constraints on the metamorphic history suggest a common metamorphism in most investigated basement domains during Upper Triassic times (Indosinian orogeny) under amphibolite-facies conditions followed by at least two thermal events during the Upper Cretaceous and the Upper Paleocene. Similar age patterns of Indosinian granites with a thermal overprint in Upper Cretateous times were reported from Phuket Islandand near Kuala Lumpur by [7]. The regional distribution of the zircon ages implies that all samples belonging to the Sibumasu terrain show the existence of at least two metamorphic events (Upper Triassic and Cretaceous) but no signs of an earlier, Middle Ordovician event. In contrast, samples originating from the Sukhothai Fold Belt (Figure 1) show evidence ofMiddle Ordovician metamorphism. The correlation of the first locality (Th 04-02) as a part of the Sukhothai Fold Belt suggested by [31] is obvious. The sample (Th 01-00) collected east of the Khlong Marui Fault reveals a more complicated tectonic relationship as it does not show typical agesignatures as expected from rocks belonging to the Sibumasu Block. A correlation with the continuation ofSukhothai Fold belt, the socalled East Malaya Block ([31]) seems to be more likely as Middle Ordovician zircons occur as in sample Th 04-02. A striking difference in both samples is the strong HT overprint during the Upper Paleoceneeast of the Khlong Marui Fault, which would agree with the tectonic interpretation of [26] who relate these locations to the Sibumasuterraine. In conclusion, the data presented above confirm the general thermo-metamorphic evolution as obtained by conventional multigrain dating ([1]) which is also reconfirmed by [26]. 


\section{Acknowledgements}

We are thankful for precise zircon SHRIMP dating by R. Armstrong (Research School of Earth Sciences, Canberra). S. Singharajwarapan guided us in the field, which we appreciate gratefully. Valuable comments and discussion on an earlier version of the manuscript made by S. Salyapongse and A.J. Barber are kindly acknowledged.

\section{References}

[1] Hansen, B.T. and Wemmer, K. (2011) The Age and Evolution of the Basement Rocks in Thailand. In: Ridd, M. F., Barber, A. J., Crow, M. J. (Eds.), The geology of Thailand, Geological Society; Distributors, North America, for trade and institutional orders, The Geological Society, c/o AIDC, London, 19-32.

[2] Heim, A. and Hirschi, H. (1939) A Section of the Mountain Range of Northwestern Siam. Ecologae Geologicae Helvetiae, 32, 1-16.

[3] Brown, G.F., Buravas, S., Charaljavanaphet, J., Jalichandra, W., Johnston, W.D., Sresthaputra, V. and Taylor, G.C. (1951) Geology Reconnaissance of the Mineral Deposits in Thailand: Geologic Investigations in Asia. United States Government Printing Office, Washington DC, 1-183.

[4] Buravas, S.C. (1952) Preliminary Notes on the Geology of Thailand. Thai Science Bulletin, 7, 7-43.

[5] Javanaphet, C. (1969) Geological Map of Thailand; Scale 1: 1,000,000 with Explanation. Department of Mineral Resources, Bangkok.

[6] Kanjanapayont, P., Thöni, M., Grasemann, B. and Edwards, M.A. (2012) Rb-Sr, Sm-Nd, and U-Pb Geochronology of the Rocks within the Khlong Marui Shear Zone, Southern Thailand. Journal of Asian Earth Sciences, 56, 263-275. http://dx.doi.org/10.1016/j.jseaes.2012.05.029

[7] Searle, M.P., Whitehouse, M.J., Robb, L.J., Ghani, A.A., Hutchison, C.S., Sone, M., Ng, S.-P., Roselee, M.H., Chung, S.-L. and Oliver, G. (2012) Tectonic Evolution of the Sibumasu-Indochina Terrane Collision Zone in Thailand and Malaysia: Constraints from New U-Pb Zircon Chronology of SE Asian Tin Granitoids. Journal of the Geological Society of London, 169, 489-500. http://dx.doi.org/10.1144/0016-76492011-107

[8] Hara, H., Kunii, M., Hisada, K.-I., Ueno, K., Kamata, Y., Charusiri, P. and Charoentitirat, T. (2012) Petrography and Geochemistry of Clastic Rocks, U-Pb Ages of Detrital Zircon from Mélange within the Inthanon Zone, Northern Thailand: Implication for Paleo-Tethys Subduction and Convergence. Acta Geoscientica Sinica, 33, Supp. 1, 15.

[9] Suensilpong, S., Burton, C.K., Mantajit, N. and Workman, D.R. (1978) Geological Evolution and Igneous Activity of Thailand and Adjacent Areas. III GEOSEA, Bangkok, 14-16.

[10] Salyapongse, S. (2002) Precambrian Rocks of Thailand. The Symposium on Geology of Thailand, Bangkok, 26-31 August 2002, 14-15.

[11] Cambell, K.V. (1974) Basement Complex. Department of Geological Sciences. Special Publication No. 1, Vol. 1, Chiang Mai University, Chiang Mai, 3-12.

[12] Dheeradilok, P. and Lumjaun, A. (1978) Metamorphic Facies Map of Southeast Asia, Scale 1:5,000,000. Geological Survey Division, Department of Mineral Resources, Bangkok.

[13] Ahrendt, H., Chonglakmani, C., Hansen, B.T. and Helmcke, D. (1991) First Results from Geochronological and Structural Investigations along the Tak-Mae Sot Highway/N-Thailand. Guidebook for the Fieldtrip No. 1. 7th Regional Conference on the Geology, Mineral and Energy Resources of SE Asia, Bangkok, 5-8 November 1991, 69-76.

[14] Ahrendt, H., Chonglakmani, C., Hansen, B.T. and Helmcke, D. (1993) Geochronological Cross Section through Northern Thailand. Journal of Southeast Asian Earth Science, 8, 207-217. http://dx.doi.org/10.1016/0743-9547(93)90022-H

[15] Mickein, A. (1997) U/Pb-, Rb/Sr- und K/Ar-Untersuchungen zur metamorphen Altersstellung des "Präkambriums" in NW-Thailand. Göttinger Arbeiten zur Geologie und Paläontologie, 1-83.

[16] Barr, S.M., Macdonald, A.S., Miller, B.V., Reynolds, P.H., Rhodes, B.P. and Yokart, B. (2002) New U-Pb and ${ }^{40} \mathrm{Ar} /$ ${ }^{39} \mathrm{Ar}$ Ages from the Doi Intharnon and Doi Suthep Metamorphic Core Complexes, Northwestern Thailand. The Symposium on Geology of Thailand, 26-31 August 2002, Bangkok, 284-294.

[17] Bachmann, G. and Grauert, B. (1986) Altersbestimmung mit 87Sr/86Sr-Ungleichgewichtsver-teilungen. Fortschritte der Mineralogie, 64, 12.

[18] Lacassin, R., Maluski, H., Leloup, P.H., Tapponnier, P., Hinthong, C., Siribakdi, K., Chuaviroj, S. and Charoenravat, A. (1997) Tertiary Diachronic Extrusion and Deformation of Western Indochina: Structural and ${ }^{40} \mathrm{Ar} /{ }^{39} \mathrm{Ar}$ Evidence from NW Thailand. Journal of Geophysical Research: Solid Earth, 102, 10013-10037. http://dx.doi.org/10.1029/96JB03831 
[19] Silver, L.T. and Deutsch, S. (1961) Uranium Lead Method on Zircons. Annals of the New York Academy of Sciences, 91, 279-283. http://dx.doi.org/10.1111/j.1749-6632.1961.tb35460.x

[20] Purdy, J.W. and Jäger, E. (1976) K-Ar Ages on Rock Forming Minerals from the Central Alps. Memorie degli Istituti di Geologia e Mineralogia dell’ Universita di Padova, 30, 3-31.

[21] Harrison, T.M., Célérier, J., Aikman, A.B., Hermann, J. and Heizler, M.T. (2009) Diffusion of ${ }^{40}$ Ar in Muscovite. Geochimica et Cosmochimica Acta, 73, 1039-1051. http://dx.doi.org/10.1016/j.gca.2008.09.038

[22] Willigers, B., Krogstad, E.J. and Wijbrans, J.R. (2001) Comparison of Thermochronometers in a Slowly Cooled Granulite Terrain: Nagssugtoqidian Orogen, West Greenland. Journal of Petrology, 42, 1729-1749. http://dx.doi.org/10.1093/petrology/42.9.1729

[23] Department of Mineral Resources (2001) Geology of Thailand. Department of Mineral Resources, Bangkok, 35-52. (In Thai)

[24] Dunning, G.R., Macdonald, A.S. and Barr, S.M. (1995) Zircon and Monazite U-Pb Dating of the Doi Inthanon Core Complex, Northern Thailand: Implications for Extension within the Indosinian Orogen. Tectonophysics, 251, 197-213. http://dx.doi.org/10.1016/0040-1951(95)00037-2

[25] Kosuwan, S. (1996) Geology of the Khanom Gneissic Complexes, Amphoe Khanom, Changwat Nakhon Si Thammarat. M.Sc. Thesis, Chulalongkorn University, Bangkok.

[26] Kawakami, T., Nakano, N., Higashino, F., Hokada, T., Osanai, Y., Yuhara, M., Charusiri, P., Kamikubo, H., Yonemura, K. and Hirata, T. (2014) U-Pb Zircon and CHIME Monazite Dating of Granitoids and High-Grade Metamorphic rocks from the Eastern and Peninsular Thailand-A New Report of Early Paleozoic Granite. Lithos, 200-201, 64-79. http://dx.doi.org/10.1016/j.lithos.2014.04.012

[27] Putthapiban, P. and Suensilpong, S. (1979) The Igneous Geology of Granitic Rocks of Hub Kapong and Hua Hin Areas-Granite Project. Department of Mineral Resources, Bangkok, 6, 1-15.

[28] Pongsapich, W., Vedchakanchana, S. and Pongprayoon, P. (1980) Petrology of the Praburi-Hin Metamorphic Complex and Geochemistry of Gneisses in It, Geological Complex and Geochemistry of Gnesses in It. Bulletin of the Geological Society of Malaysia, 12, 55-74.

[29] Bignell, J.D. and Snelling, N.J. (1977) K-Ar Ages on Some Basic Igneous Rocks from Peninsula Malaysia and Thailand. Bulletin of the Geological Society of Malaysia, 8, 89-93.

[30] Beckinsale, R.D., Suensilpong, S., Nakapadungrat, S. and Walsh, J.N. (1979) Geochronology and Geochemistry of Granite Magmatism in Thailand in Relation to a Plate Tectonic Model. Journal of the Geological Society, 136, 529-537. http://dx.doi.org/10.1144/gsigs.136.5.0529

[31] Metcalfe, I. (2013) Gondwana Dispersion and Asean Accretion: Tectonic and Palaeo-Geographic Evolution of Eastern Tethys. Journal of Asian Earth Sciences, 66, 1-33. http://dx.doi.org/10.1016/j.jseaes.2012.12.020

[32] Paces, J.B. and Miller, J.D. (1993) Precise U-Pb Ages of Duluth Complex and Related Mafic Intrusions, Northeastern Minnesota: Geochronological Insights to Physical, Petrogenic, Paleomagnetic and Tectonomagmatic Processes Associated with the 1.1 Ga Midcontinent Rift System. Journal of Geophysical Research: Solid Earth, 98, 13997-14013. http://dx.doi.org/10.1029/93JB01159

[33] Williams, I.S. (1998) U-Th-Pb Geochronology by Ion Microprobe. In: McKibben, M.A., Shanks III, W.C. and Ridley, W.I., Eds., Applications of Microanalytical Techniques to Understanding Mineralizing Processes, 7, 1-35.

[34] Ludwig, K.R. (2000) SQUID 1.00, A User’s Manual. Berkeley Geochronology Center Special Publications, Berkeley, 2.

[35] Ludwig, K.R. (1998) On the Treatment of Concordant Uranium-Lead Ages. Geochimica et Cosmochima Acta, 62, 665676. http://dx.doi.org/10.1016/S0016-7037(98)00059-3

[36] Ludwig, K.R. (2011) Isoplot: A Plotting and Regression Program for Radiogenic Isotope Data. Version 4.13-March 2011. US Geological Survey Open-File Report, 445.

[37] Frei, R. and Kamber, B.S. (1995) Single Mineral Pb-Pb Dating. Earth and Planetary Science Letters, 129, $261-268$. http://dx.doi.org/10.1016/0012-821X(94)00248-W

[38] Dahl, P.S. and Frei, R. (1998) Step-Leach Pb-Pb Dating of Inclusion-Bearing Garnet and Staurolite, with Implications for Early Proterozoic Tectonism in the Black Hills Collisional Orogen, South Dakota, United States. Geology, 26, 111114. http://dx.doi.org/10.1130/0091-7613(1998)026<0111:SLPPDO >2.3.CO;2

[39] Todt, W., Cliff, R.A., Hanser, A. and Hofmann, A.W. (1993) Recalibration of NBS Lead Standards Using a $207 \mathrm{~Pb} / 205$ Pb Double Spike. Terra Abstracts, 5, 396.

[40] DePaolo, D.J. (1981) Neodymium Isotopes in the Colorado Front Range and Crust-Mantle Evolution in the Proterozoic. Nature, 291, 193-196. http://dx.doi.org/10.1038/291193a0

[41] Tütken, T., Eisenhauer A., Wiegand, B. and Hansen, B.T. (2002) Glacial-Interglacial Cycles in Sr and Nd Isotopic 
Composition of Artic Marine Sediments: Changes in Sediment Provenance Triggered by the Barents Sea Ice Sheet. Marine Geology, 182, 351-372. http://dx.doi.org/10.1016/S0025-3227(01)00248-1

[42] Ridd, M.F., Barber, A.J. and Crow, M.J. (2011) Introduction to the Geology of Thailand. In: Ridd, M.F., Barber, A.J. and Crow, M.J., Eds., The Geology of Thailand, The Geological Society, c/o AIDC, London, Williston, 1-18.

[43] Wemmer, K. and Ahrendt, H. (1997) Comparative K-Ar and Rb-Sr Age Determinations of Retrograde Processes on Rocks from the KTB Deep Drilling Project. Geologische Rundschau, 86, S272-S285.

http://dx.doi.org/10.1007/PL00014660

\section{Appendix}

\section{SHRIMP U/Pb Dating}

All zircons were mounted in epoxy resin at the Research School of Earth Sciences (RSES, Canberra), together with the RSES reference zircons FC1 and SL13. Photomicrographs in transmitted and reflected light were taken of all zircons. These, together with SEM CL and BSE images, respectively, were used to decipher the internal structures of the sectioned grains and to target specific areas within the accessories (i.e. metamorphic rims or inherited cores) using a 4 - 6 nA primary $\mathrm{O}_{2}$ ion beam with an c. $25 \mu \mathrm{m}$ diameter spot. The U-Pb zircon analyses were carried out in a single session on the SHRIMP II. For the zircon calibration the $\mathrm{Pb} / \mathrm{U}$ ratios were normalized relative to a value of 0.1859 for the ${ }^{206} \mathrm{~Pb} /{ }^{238} \mathrm{U}$ ratio of FC1 reference zircons, equivalent to an age of 1.099 $\mathrm{Ga}$ ([32]). U and Th concentrations were determined relative to the SL13 standard. The error in the standard calibration was $0.25 \%$ on the SHRIMP II. To reduce any isobaric interference on mass ${ }^{204} \mathrm{~Pb}, 50 \%$ energy filtering was applied to the secondary beam. Standard analyses were carried out, producing a $2 \sigma$ error of the (weighted) mean $\mathrm{Pb} / \mathrm{U}$ calibration of $0.64 \%$. Correction for $\mathrm{Th} / \mathrm{U}$ fractionation was calculated directly using the method of [33], and all other data reduction utilized the SQUID software of [34].Uncertainties given for individual analyses (ratios and ages) are at the $1 \sigma$ level; however, uncertainties in any calculated weighted mean ages or Concordia ages ([35]) are reported as 95\%confidence limits (unless stated otherwise) and include the uncertainties in the standard calibrations where appropriate. Concordia plots, regressions and weighted mean age calculations were carried out using Isoplot 4.13 ([36]).

\section{Pb Stepwise Leaching (PbSL)}

$\mathrm{Pb} / \mathrm{Pb}$ stepwise leaching experiments (PbSL) were applied to one garnet separate. Slightly modified procedures of [37] were applied, in an effort to date the formation age of this mineral phase. Mineral separates were obtained by standard techniques using jaw crusher and sieve kit. The 150 - $250 \mu \mathrm{m}$ sieve fraction was then purified by handpicking followed by repeated rinsing in deionized water and $200 \mathrm{mg}$ of this materials was transferred to $7 \mathrm{ml}$ Savillex1 screw-cap beakers for step-leaching. Successive $120^{\circ} \mathrm{C}$ acid leach steps (five in total) involving various concentrations of $\mathrm{HBr}$, to extract $\mathrm{Pb}$ selectively from the phases. The leaching schemes are given in table 5. Purified $\mathrm{Pb}$ extracts were mounted on Re filaments and $\mathrm{Pb}$ isotopic ratios were determined by thermal ionization mass spectrometry (TIMS). A similar PbSL technique was previously applied to garnet in metapelite ([38]). Fractionation for $\mathrm{Pb}$ was controlled by repeated analysis of the NBS 981 standard and amounted to $0.103 \pm$ $0.007 \%$ per a.m.u. $(2 \sigma ; \mathrm{n}=5)$ relative to the values proposed by [39]. Procedural blanks for Pb remained below $87 \mathrm{pg}$. The amount insignificantly affects the isotopic data for the samples. 
Scientific Research Publishing (SCIRP) is one of the largest Open Access journal publishers. It is currently publishing more than 200 open access, online, peer-reviewed journals covering a wide range of academic disciplines. SCIRP serves the worldwide academic communities and contributes to the progress and application of science with its publication.

Other selected journals from SCIRP are listed as below. Submit your manuscript to us via either submit@scirp.org or Online Submission Portal.
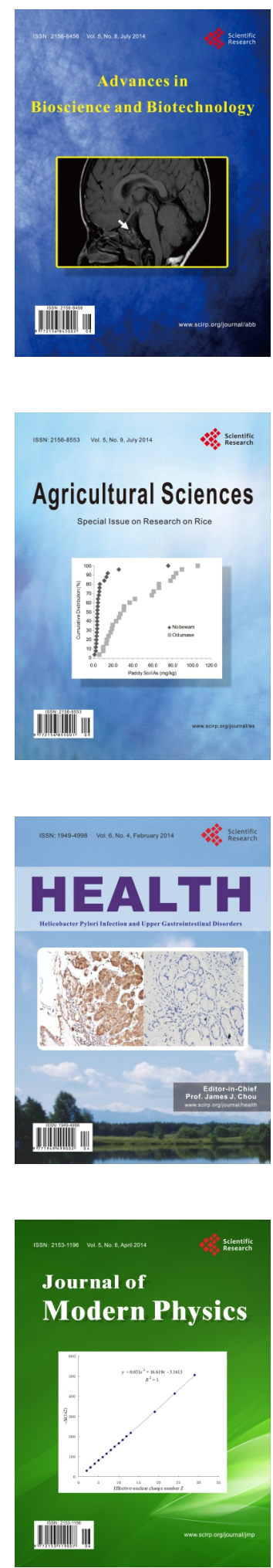
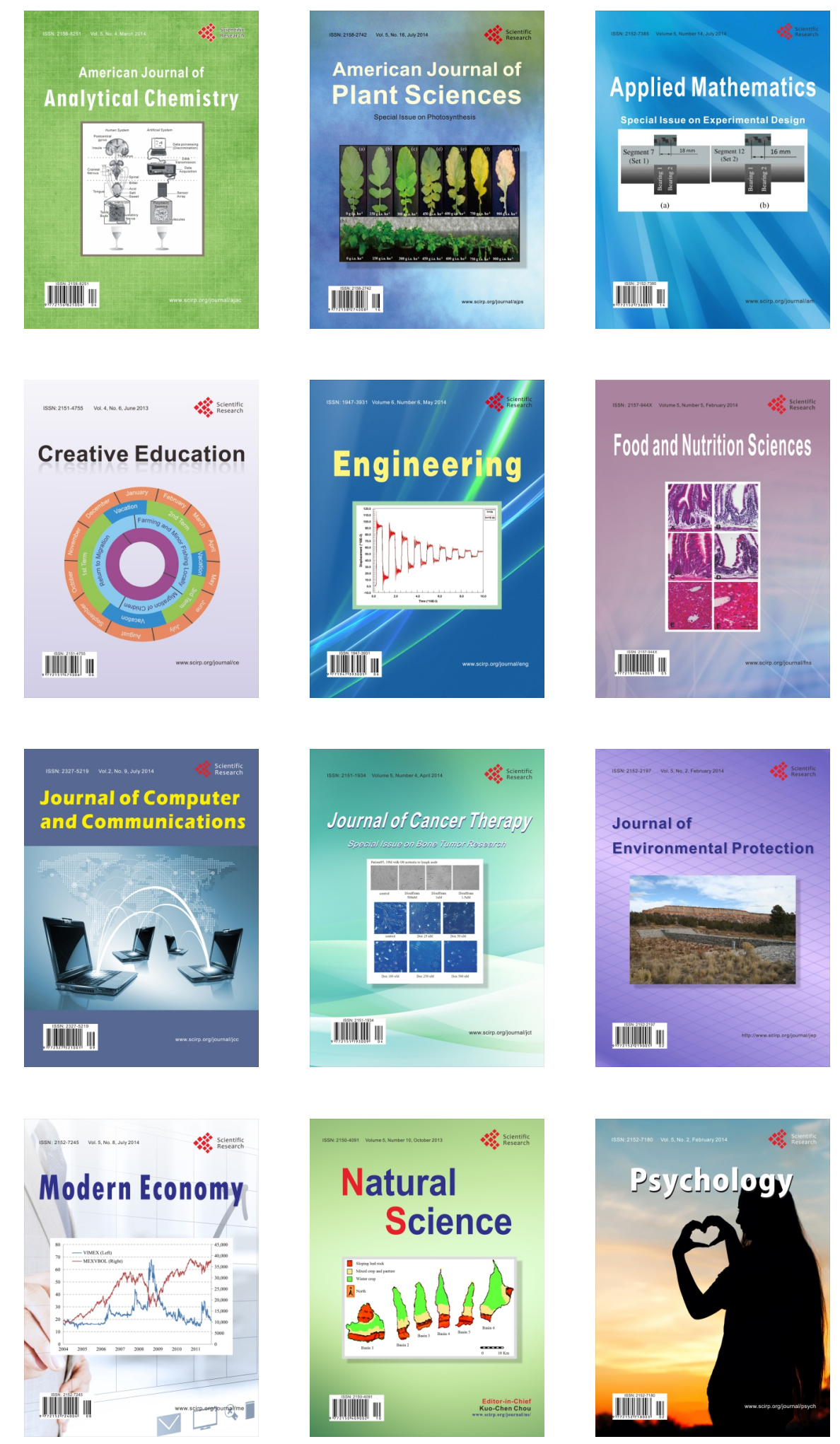